\title{
Model Test Research on Excavation Method of Large-span and Shallow-buried Bias Tunnel in Cobble and Gravelly Soil Layer
}

\author{
Tang Jinsong ${ }^{\mathrm{a}}$, Xiong Baolin ${ }^{\mathrm{b}}$ and Liu Chiguang ${ }^{\mathrm{c}}$ \\ (School of Civil Engineering, Shijiazhuang Tiedao University, Shijiazhuang 050043, China) \\ atangjssjz@163.com, bxiongbao77@163.com, liuchiguang1977@163.com
}

\begin{abstract}
Keywords: cobble and gravelly soil; bias tunnel; model test; excavation method; large-span; shallow-buried

Abstract. In order to select reasonable excavation method of tunnel engineering in complex conditions, model test was done for large-span and shallow-buried bias tunnel in cobble and gravelly soil layer, the reasonable excavation scheme was determined by analysis of tunnel surrounding rock and internal support stress after excavation. The bench cut method and the four-partial excavation method with temporary diaphragm in top heading are adopted to excavate model tunnel. It is resulted that the reasonable excavation method is the four-partial excavation method with temporary diaphragm in top heading. The large-span and shallow-buried bias tunnel in the cobble and gravelly soil layer is excavated from the shallow excavation site and has less deformation than that of the deep excavation. Model test can correctly reflect some changes of the real formation, and some problems in the construction process can be prevented in advance.
\end{abstract}

\section{Introduction}

Because too many uncertain factors exist in geotechnical and underground engineering, the uncertain factors cannot be predicted only by experience. There is little study on the constitutive relationship of rock and soil ${ }^{[1-3]}$. Numerical simulation and theoretical research methods can not accurately reflect the nature of the project. The similar model test can not only reflect constitutive relation of rock and soil in the real engineering, but also can simulate the impact of the structure under the tunnel different excavation method ${ }^{[4-5]}$. The direct data is monitored in different location of test models. These provide convenience to study the stability of tunnel structure, avoid adverse factors and guide the construction site ${ }^{[6]}$.

\section{General engineering situation}

Maoshan Xi tunnel is the down line for Maoshan tunnel with a total length of $582 \mathrm{~m} .80 \%$ section of the entire tunnel is below $50 \mathrm{~m}$ of the tunnel buried depth. The most shallow depth is less than $2 \mathrm{~m}$. Maoshan Xi tunnel belongs to the large-span shallow-buried highway tunnel. The most part of the Maoshan Xi tunnel is across the cobble and gravelly soil layer which is broken and relatively poor in the stability.

\section{Model conceptual design}

Model establishing. According to the geometric similarity ratio, the tunnel prototype is reduced to model test. The length, width and height of the model size used in the model test are $3000 \mathrm{~mm} \times 1500 \mathrm{~mm} \times 2000 \mathrm{~mm}$, as shown in Fig. 1 . In order to be more intuitive to observe the tunnel excavation process and trend, the front of the indoor model tank is built with $15 \mathrm{~mm}$ thick tempered glass. In order to prevent damage of toughened glass by the earth pressure in the filling process, lateral tempered glass is supported by thin steel plate. Model tunnel mouth location from the left and right side wall is $1.3 \mathrm{~m}$, lower edge from trough bottom $1.18 \mathrm{~m}$, span $0.43 \mathrm{~m}$, height $0.3 \mathrm{~m}$. To facilitate positioning, outer wall of the tunnel is sprinkled with lime powder, as shown in Fig. 2. 


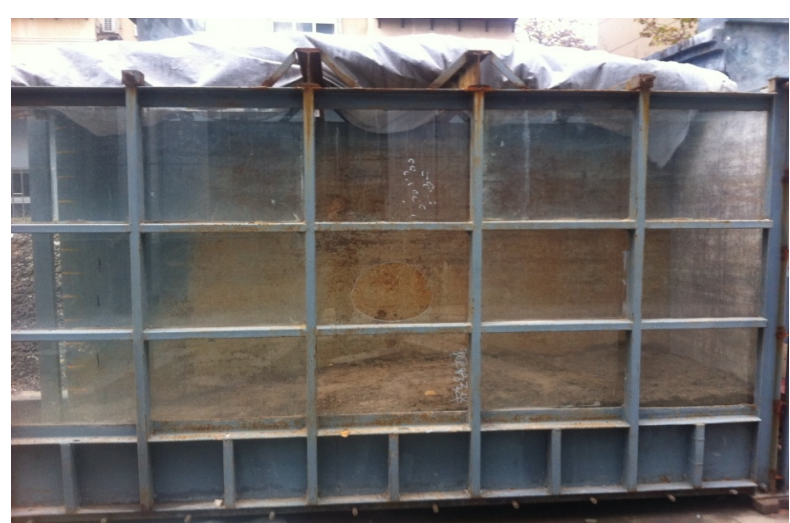

Fig. 1 Model slot

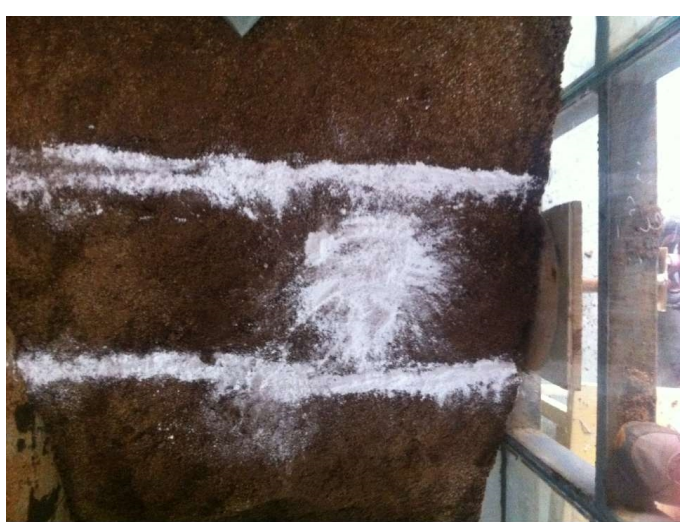

Fig. 2 Boundary line of tunnel excavation

Measuring point arrangement. A series of monitoring points are arranged in the middle section of the model test. The purpose of the test determines the layout of the monitoring elements. The main observation items of the model include the displacement of the surrounding rock, the displacement around the tunnel, the radial pressure and the support stress, etc. On the monitoring section, the radial pressure measuring lines of vault, arch shoulder and arch foot are 5, as shown in Fig. 3 and the measuring point of the tunnel displacement is shown in Fig. 4.

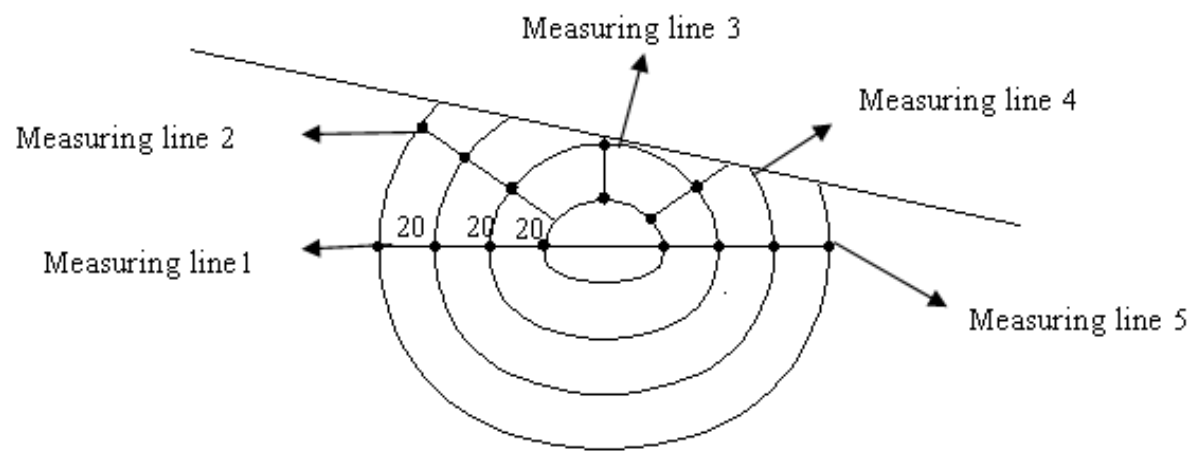

Fig. 3 Arrangement of radial pressure measuring points at hole

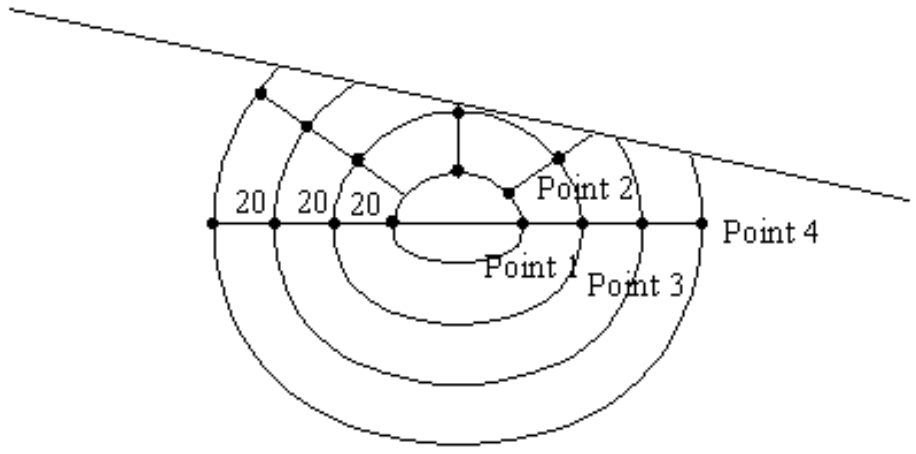

Fig. 4 Arrangement of measuring points of hole displacement

Excavation method of the model test. The bench cut method and the four-partial excavation method with temporary diaphragm in top heading are adopted in this model test.

The bench cut method is that the upper step of the tunnel model is firstly excavated by using self-made Luoyang shovel and excavation length is $5 \mathrm{~cm}$. The excavation process should be gentle to prevent the collapse of the tunnel model. The lining is immediately established after the excavating completion of the upper step of the tunnel model. The down step is excavated and the lining is constructed 10 minutes later after completing the upper step. Then, the initial support is established. According to the above construction sequence, cycle excavation is until perforation.

The excavating sequence of the four-partial excavation method with temporary diaphragm in top heading is that the first is the upper right section, the second is primary support and temporary support on the upper right section, the third is the upper left section, the fourth is primary support on the upper left section on the section, the fifth is the down right section and inverted arch, the sixth is primary support of the down right section, the seventh is the removal of temporary support, the eighth is the 
down left section and inverted arch and the last is primary support of the down left section and inverted arch.

\section{Analysis and comparison of test results}

\section{Internal displacement analysis of surrounding rock}

1 Analysis of the horizontal displacement of each measuring point with the excavation step on the left foot monitoring line 1.

The relation between the horizontal displacement of each measuring point and the excavation step on the monitoring line 1 of the left foot of the tunnel is shown in Fig. 5 and Fig. 6.

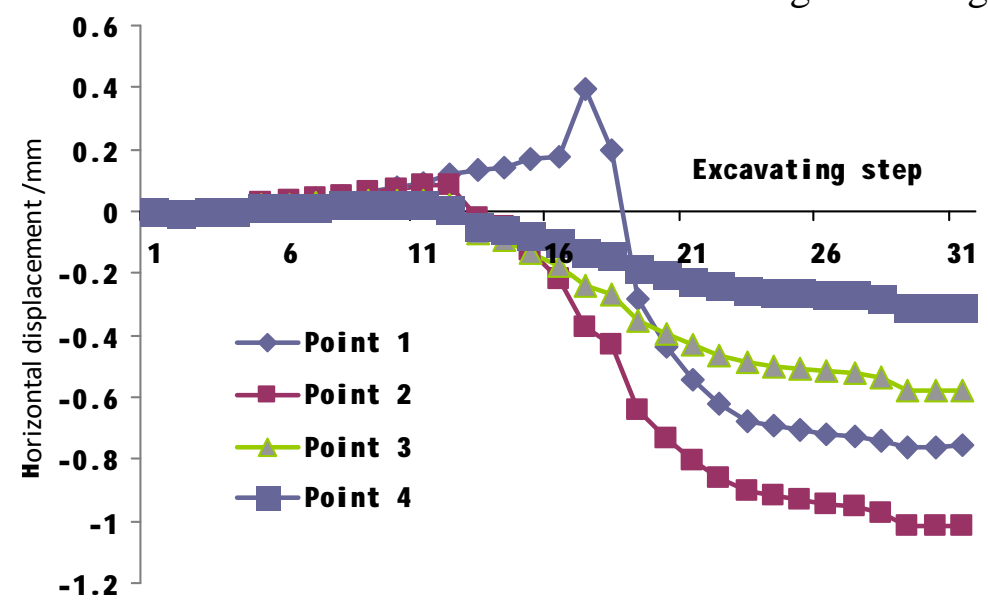

Fig. 5 Relationship between each measuring point on the monitoring line 1 on the bench cut method

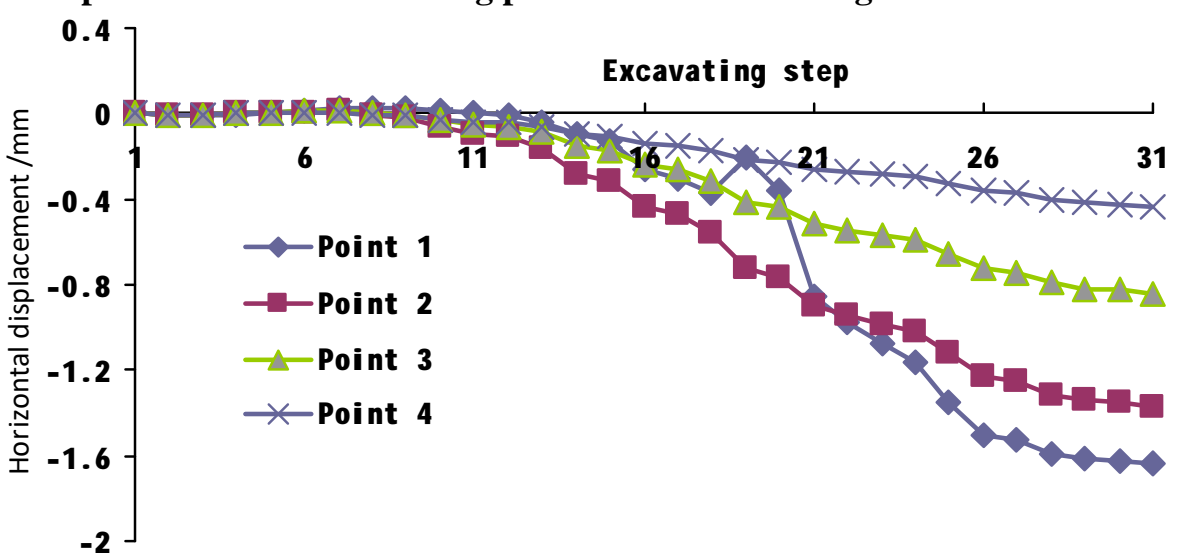

Fig. 6 Relationship between each measuring point on the monitoring line 1 on the four-partial excavation method with temporary diaphragm in top heading

From Fig. 5 and Fig. 6 can be drawn: in the bench cut method monitoring points 1 of monitoring section appear relatively large positive in excavation. Monitoring points 1 happen to the left shift, moving forward along with the excavation constantly face. Horizontal displacement of 4 monitoring points in the monitoring line between the four-partial excavation method with temporary diaphragm in top heading and the bench cut method are different. Horizontal displacement value of the four-partial excavation method with temporary diaphragm in top heading is greater than that of the bench cut method.

2 Analysis of the vertical displacement of each measuring point with the excavation step on the left waist monitoring line 2

The vertical displacements of each measuring point with the excavation step on the left waist monitoring line 2 are respectively shown in Fig. 7 and Fig. 8 by the bench cut method and the four-partial excavation method with temporary diaphragm in top heading. 


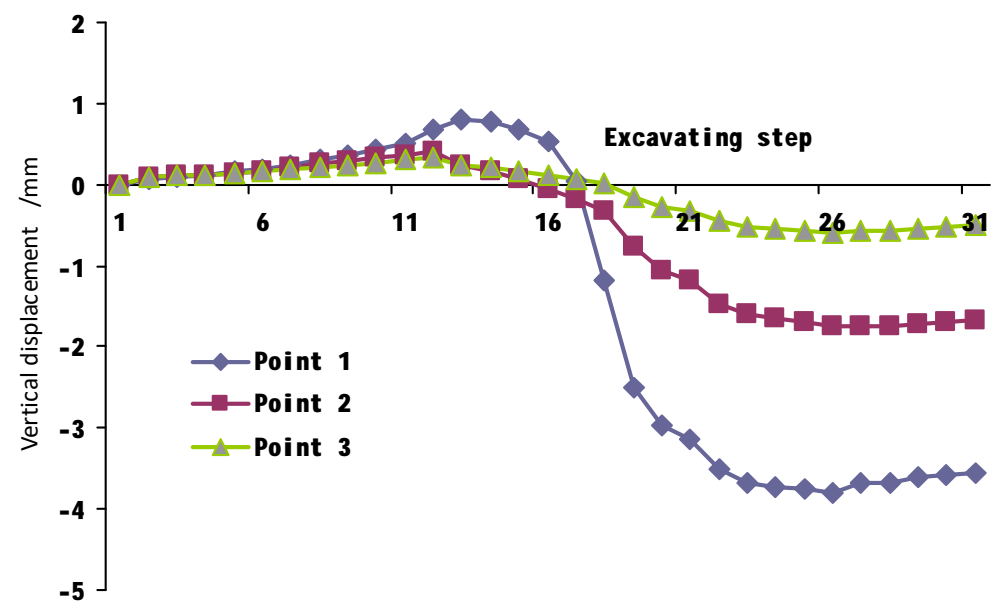

Fig. 7 Relationship between the vertical displacements of each measuring point with the excavation step by the bench cut method

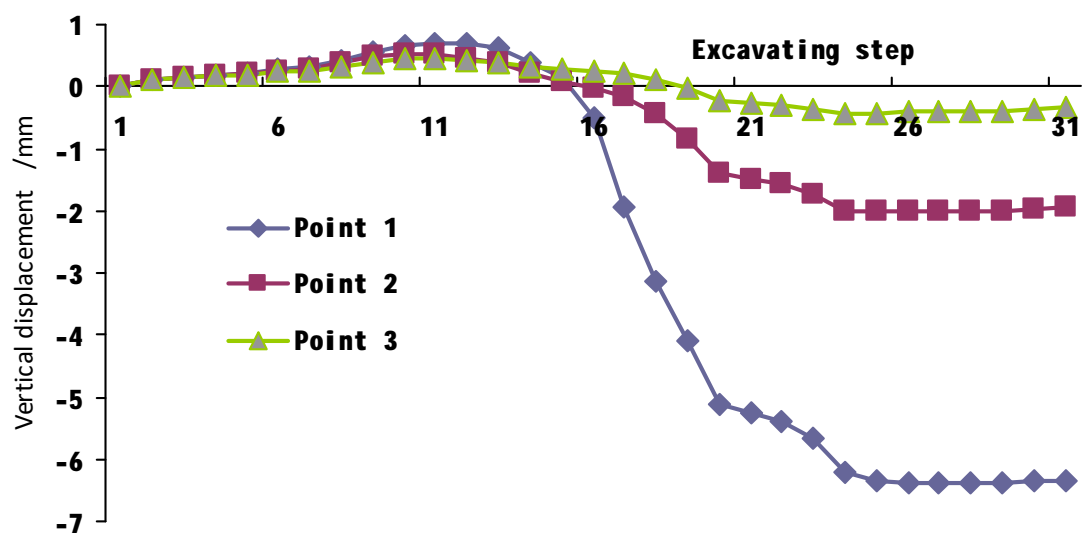

Fig. 8 Relationship between the vertical displacements of each measuring point with the excavation step by the four-partial excavation method with temporary diaphragm in top heading

Fig. 7 and Fig. 8 show that excavation step gradually increases to the location of the monitoring sections, vertical displacement of the bench cut method and the four-partial excavation method with temporary diaphragm in top heading downward change rapidly. The vertical displacement of the monitoring points to the steady state, but the vertical displacement of 3 monitoring points in the monitoring line are different.

3 Analysis of the vertical displacement of each measuring point with the excavation step on the arch top under the monitoring line 3

The vertical displacements of each measuring point with the excavation step on the arch top under the monitoring line 3 are respectively shown in Fig. 9 and Fig. 10 by the bench cut method and the four-partial excavation method with temporary diaphragm in top heading.

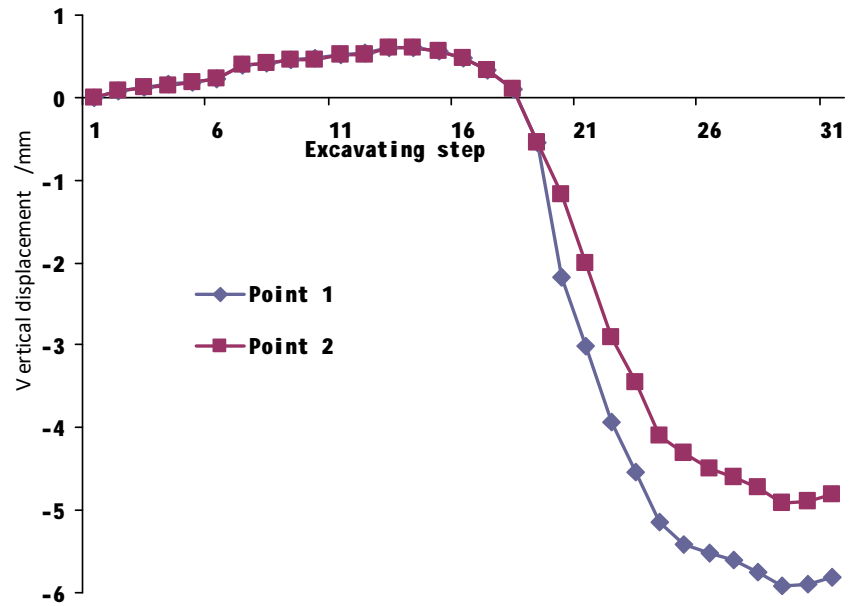

Fig. 9 Relationship between the vertical displacements of each measuring point under the monitoring line 3 with the excavation step by the bench cut method 


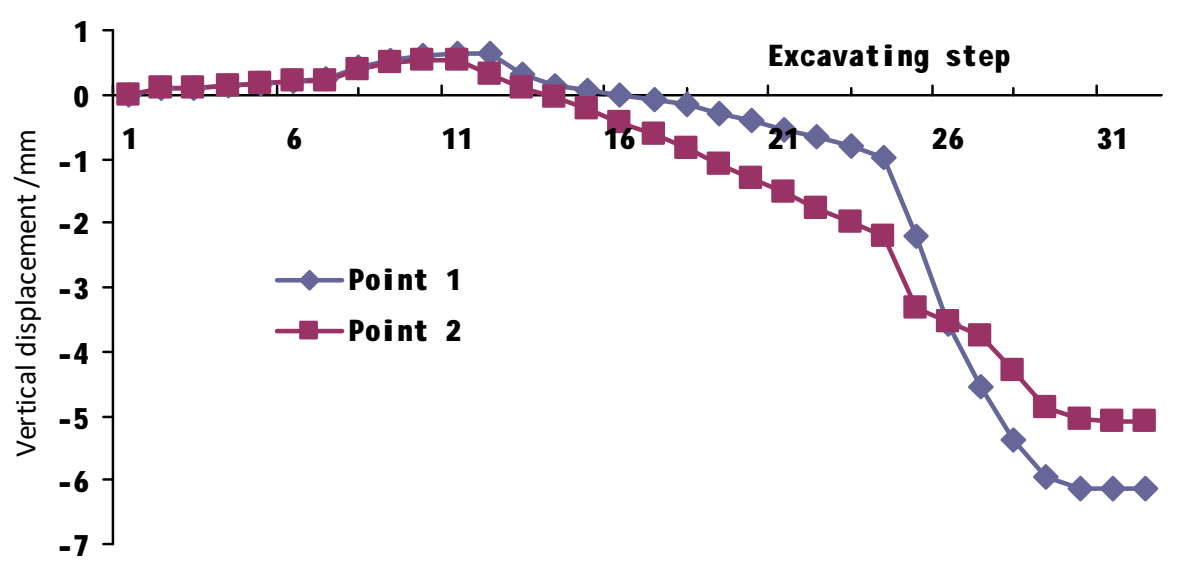

Fig. 10 Relationship between the vertical displacements of each measuring point under the monitoring line 3 with the excavation step by the four-partial excavation method with temporary diaphragm in top heading

From Fig. 9 and Fig. 10, two monitoring points of the tunnel vault bench position line have upper deformation during the excavation step 5 18 for deformation in the bench cut method. With the increasing of excavation step, the vertical displacement of two monitoring points in the tunnel vault performance line 3 is downward on the deformation. The vertical displacement of the tunnel vault performance half broken face supporting excavation during step 5 14 uplifts.

4 Analysis of the vertical displacement of each measuring point with the excavation step on the right waist under the monitoring line 4

The vertical displacements of each measuring point with the excavation step on the arch top under the monitoring line 4 are respectively shown in Fig. 11 and Fig. 12 by the bench cut method and the four-partial excavation method with temporary diaphragm in top heading.

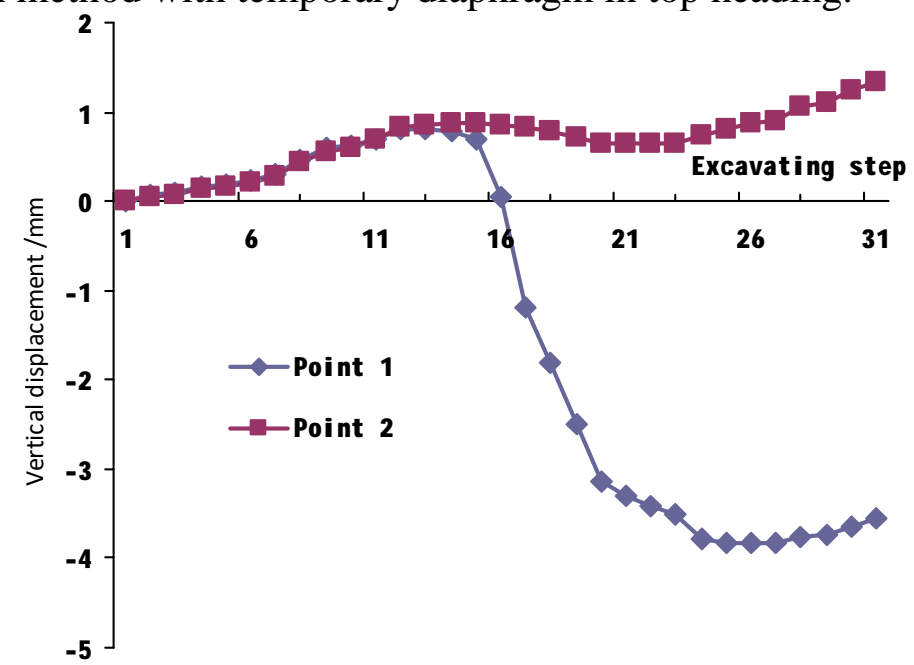

Fig. 11 Relationship between the vertical displacements of each measuring point under the monitoring line 4 with the excavation step by the bench cut method

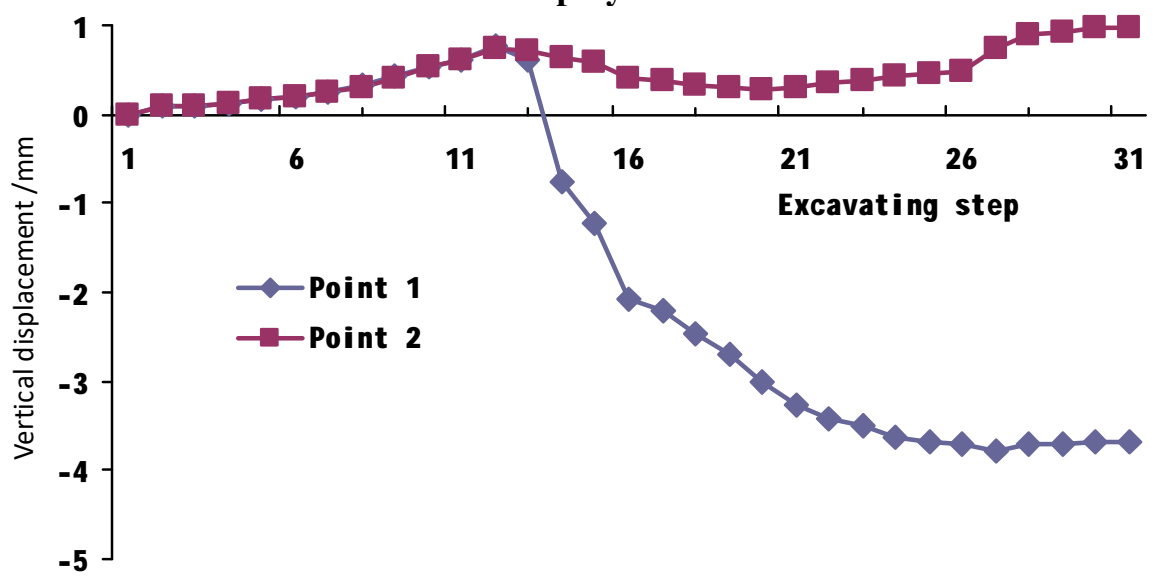

Fig. 12 Relationship between the vertical displacements of each measuring point under the monitoring line 4 with the excavation step by the four-partial excavation method with temporary diaphragm in top heading 
From Fig. 11 and Fig. 12 can be seen that the vertical displacement on the right side of tunnel arch between the bench cut method and the four-partial excavation method with temporary diaphragm in top heading is basically consistent with the monitoring points. In two different conditions of tunnel excavation method on the right side of the waist line 4 on the 2 monitoring in the excavation process of the whole deformation upward uplift, which is due to the bias strata above the tunnel, but also the tunnel excavation is also not stable trend.

5 Analysis of the horizontal displacement of each measuring point with the excavation step on the right foot arch under the monitoring line 5

The horizontal displacements of each measuring point with the excavation step on the right foot arch under the monitoring line 5 are respectively shown in Fig. 13 and Fig. 14 by the bench cut method and the four-partial excavation method with temporary diaphragm in top heading.

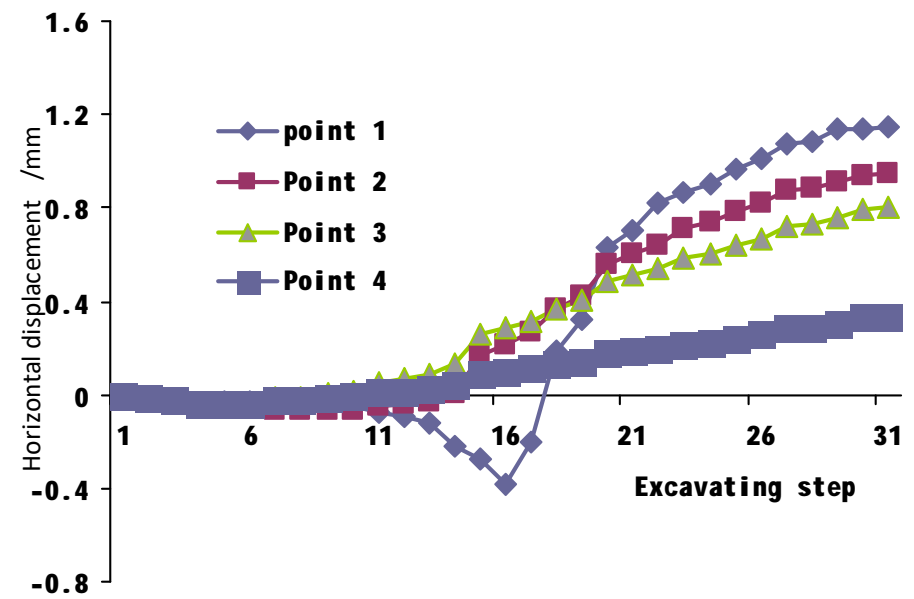

Fig. 13 Relationship between the horizontal displacements of each measuring point under the monitoring line 5 with the excavation step by the bench cut method

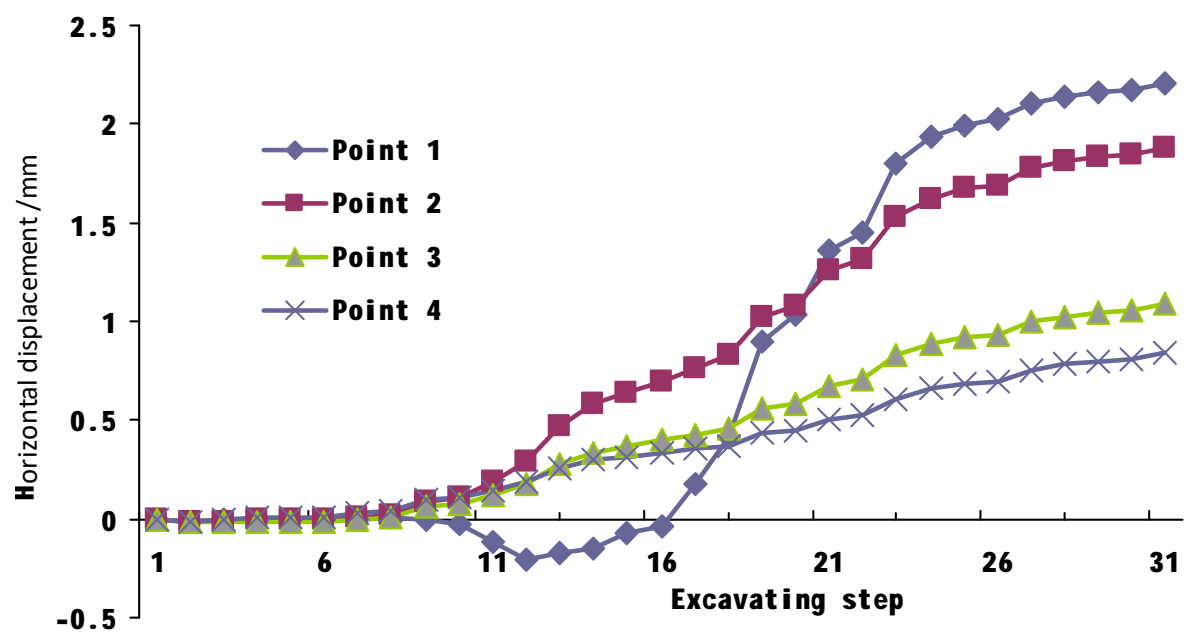

Fig. 14 Relationship between the horizontal displacements of each measuring point under the monitoring line 5 with the excavation step by the four-partial excavation method with temporary diaphragm in top heading

From Fig. 13 and Fig. 14 can be seen that the right side of the arch foot in the monitoring section monitoring on line 5 of all monitoring points in the excavation process are left for deformation between the bench cut method and the four-partial excavation method with temporary diaphragm in top heading.

6 Settlement of the vault and horizontal convergence of tunneling

Settlement of the vault and horizontal convergence of tunneling monitoring section under the bench cut method and the four-partial excavation method with temporary diaphragm in top heading is shown in table 1. 
Table 1 Settlement of the vault and horizontal convergence in the different excavating method

\begin{tabular}{ccc}
\hline Case & Settlement of the vault $/ \mathrm{mm}$ & Horizontal convergence $/ \mathrm{mm}$ \\
\hline $\begin{array}{c}\text { The bench cut method } \\
\text { method with temporary } \\
\text { diaphragm in top heading }\end{array}$ & 5.92 & 1.91 \\
\hline
\end{tabular}

It can be seen from table 1 that the settlement of the tunnel vault is larger than the horizontal convergence of the tunnel during the construction of large-span and shallow-buried bias tunnel under two different construction methods, namely, the bench cut method and the upper half section temporary support method. Under the four-partial excavation method with temporary diaphragm in top heading, the settlement of the vault of the tunnel has little difference with the settlement of the bench cut method, but the horizontal convergence value of the tunnel by the four-partial excavation method with temporary diaphragm in top heading is greater than that of the bench cut method.

\section{Radial pressure analysis of surrounding rock}

When the model tunnel is excavated, the pressure of surrounding rock of tunnel monitoring section is shown in Table 2 and table 3 under the bench cut method and the four-partial excavation method with temporary diaphragm in top heading.

Table 2 Pressure of the surrounding rock under the bench cut method /MPa

\begin{tabular}{ccccc}
\hline \multirow{2}{*}{ Line number } & \multicolumn{4}{c}{ Monitoring point } \\
\cline { 2 - 5 } & 1 & 2 & 3 & 4 \\
\hline Line 1 & 0.236 & 0.314 & 0.284 & 0.230 \\
Line 2 & 0.087 & 0.082 & 0.002 & $/$ \\
Line 3 & 0.065 & 0.067 & $/$ & $/$ \\
Line 4 & 0.154 & 0.162 & $/$ & $/$ \\
Line 5 & 0.220 & 0.271 & 0.244 & 0.226 \\
\hline
\end{tabular}

Table 3 Pressure of the surrounding rock under the four-partial excavation method with temporary diaphragm in top heading /MPa

\begin{tabular}{ccccc}
\hline \multirow{2}{*}{ Line number } & \multicolumn{4}{c}{ Monitoring point } \\
\cline { 2 - 5 } & 1 & 2 & 3 & 4 \\
\hline Line 1 & 0.173 & 0.181 & 0.192 & 0.185 \\
Line 2 & 0.032 & 0.031 & 0.030 & $/$ \\
Line 3 & 0.196 & 0.118 & $/$ & $/$ \\
Line 4 & 0.029 & 0.021 & $/$ & $/$ \\
Line 5 & 0.159 & 0.162 & 0.169 & 0.226 \\
\hline
\end{tabular}

From table 2 and 3 can be seen that measuring position 2 in the monitoring of line 1 happens maximum of the surrounding rock pressure under the bench cut method and the four-partial excavation method with temporary diaphragm in top heading. The excavation of the tunnel has a great influence on the left arch of the tunnel and has little influence on the left shoulder of the tunnel. Moreover, the pressure of the surrounding rock caused by the bench cut method is larger than that the four-partial excavation method with temporary diaphragm in top heading.

\section{Support stress analysis of tunnel}

In the course of tunnel excavation, the change of lining stress is very important to study the stability of tunnel. Therefore, the monitoring of lining stress during tunnel excavation is a must. A total of 8 monitoring points have been set up to measure the support stress. The stress values under the two excavation schemes are shown in table 4.

From the table 4, it can be seen that the bench cut method in the vault supporting stress appears minimum, maximum right shoulder, second left foot arch of the tunnel. due to the impact of bias, the excavation process of deep buried side rock may be shallow lateral extrusion, causing shallow lateral deformation of the initial support. the support should be based on the ratio of the stress in the deep branch. The support stress of the right abutment is the smallest and the maximum at the bottom of the tunnel under the four-partial excavation method with temporary diaphragm in top heading. 
Table 4 Support stress of two excavating methods /MPa

\begin{tabular}{ccc}
\hline & \multicolumn{2}{c}{ Excavating method } \\
\cline { 2 - 3 } Monitoring position & The bench cut method & $\begin{array}{c}\text { The four-partial excavation } \\
\text { method with temporary } \\
\text { diaphragm in top heading }\end{array}$ \\
\hline Arch top & 0.226 & 0.212 \\
Left shoulder & 2.210 & 0.031 \\
Right shoulder & 2.838 & 0.0275 \\
Left waist & 1.121 & 0.171 \\
Right waist & 0.919 & 0.159 \\
Left foot arch & 1.014 & 0.037 \\
Right foot arch & 1.448 & 0.037 \\
Tunneling bottom & 0.247 & 0.484 \\
\hline
\end{tabular}

\section{Conclusions}

It is resulted that the reasonable excavation method is the four-partial excavation method with temporary diaphragm in top heading. The reason is that this method perfectly controls settlement of surrounding rocks and ensures the construction safety.

The large-span shallow-buried bias tunnel in the cobble and gravelly soil layer is excavated from the shallow excavation site and has less deformation than the deep excavation. The advance pre-support of the arch is made ahead of the excavation of the tunnel.

The excavation of the cobble and gravelly soil layer reduces the number of excavation face and completes the initial support in a timely manner. Model test can correctly reflect some changes of the real formation, and some problems in the construction process can be prevented in advance.

\section{Acknowledgements}

This work was financially supported by the Hebei Natural Science Foundation (E2014210021).

\section{References}

[1] Femando R. Ground subsidence due to a shallow tunnel in dense sandy gravel[J]. Journal of Geotechnical and Geoenvironmental Engineering, 2002, 128(5): 426-434.

[2] Li Xiao, Liao Qiulin, He Jianming. In-site tests and Stochastic structural model of rack and soil aggregate in the three gorges reservoir area[J]. International Journal of Rock Mechanics and Mining Sciences, 2004, 41(3): 494-462.

[3] WEN Chuanyuan. Exploration on comprehensive simulation system and comprehensive similarity theory[J]. System simulation technology, 2005, 1(1):1-7.

[4] ZHU He-hua, XU Qian-wei, FH De-ming, et al. Study on design principle of shield machine applicable to different strata [J]. Rock and Soil Mechanics, 2006, 9(9): 1437-1441.

[5] LI Xiao, LIAO Qiulin, HE Jianming, CHEN Jian. Study on in-situ tests of mechanical characteristics on soil-rock aggregate[J]. Chinese Journal of Rock Mechanics and Engineering, 2007, 26(12): 2377-2384.

[6] Gao Mingzhong, Zhang Ru, Wang Man. The mechanism of ground subsidence induced by EPB tunneling in sand and cobble stratum[J]. 2013, 12(13): 447-453. 